\title{
Testing for Cointegration in a Double-LSTR Framework
}

\author{
Claudia Grote and Philipp Sibbertsen
}

\begin{abstract}
This paper investigates the finite-sample properties of the smooth transition-based cointegration test proposed by Kapetanios et al. (2006) when the data generating process under the alternative hypothesis is a globally stationary second order LSTR model. The provided procedure describes an application to long-run equilibrium relations involving real exchange rates with symmetric behaviour. We utilise the properties of the double LSTR transition function that features unit root behaviour within the inner regime and symmetric behaviour in the outer regimes. Hence, under the null hypothesis we imply no cointegration and globally stationary D-LSTR cointegration under the alternative. As a result of the identification problem the limiting distribution derived under the null hypothesis is non-standard. The Double LSTR is capable of producing three-regime TAR nonlinearity when the transition parameter tends to infinity as well as generating exponential-type nonlinearity that closely approximates ESTR nonlinearity. Therefore, we find that the Double LSTR error correction model has power against both of these alternatives. JEL codes: $C 12$, C32
\end{abstract}

Key words: Cointegration tests, LSTR, Monte carlo simulation, Nonlinear error correction

Claudia Grote

Institute of Statistics, Faculty of Economics and Management, Leibniz University Hannover, D30167 Hannover, Germany e-mail: grote@ statistik.uni-hannover.de

Philipp Sibbertsen

Institute of Statistics, Faculty of Economics and Management, Leibniz University Hannover, D30167 Hannover, Germany e-mail: sibbertsen@statistik.uni-hannover.de

This paper is dedicated to Sigfried Heiler, a most inspiring econometrician. We are indebted to Philip Bertram for helpful comments and remarks. 


\section{Introduction}

Ever since the concept of cointegration has been introduced by Granger (1981) and Engle and Granger (1987), research on cointegrated time series has experienced a broad expansion. Yet it is still developing and of great importance for economic applications such as exchange rates and equity indices, cf. Maki (2012) or Zhang (2013). One of the latest research branches is the extension of cointegration to nonlinear dynamics and regime-switching error correction mechanisms. With regard to the nonlinear cointegration literature, a distinction is drawn between time-varying cointegration on the one hand, cf. Bierens and Martins (2010) or Shi and Phillips (2012), and nonlinear adjustment processes on the other hand. Recently, the latter has been of major interest implying unsteady and unproportional correction of the disequilibrium error which is why particular attention has been directed towards testing the existence of nonlinearities, cf. Kapetanios et al. (2006) henceforth KSS, or Kiliç (2011). Thus, due to the ability to incorporate smooth dynamic adjustment via smooth transition (STR) functions, STR-models are widely applied for modelling the disequilibrium error.

Regime-switching cointegration can be considered as an approach that deals with the combination of nonlinearities and nonstationarities. It combines cointegration as the global problem and nonlinearity as the local problem, cf. Balke and Fomby (1997). Depending on the specification, the underlying testing problem can be formulated as either unit root or linearity against STR cointegration, see also Dufrénot et al. (2006). First approaches suggested a null hypothesis of no nonlinear adjustment in a linear cointegration framework and consequently based inference on a linear error correction model (ECM), cf. Seo (2004) or Nedeljkovic (2011). Among others KSS established appropriate theoretical foundations for inference based on a nonlinear ECM. In accordance with these authors it is reasonable to utilise a test that is designed to have power against the alternative of nonlinear dynamic adjustment. The reason why research focus has come to allow nonlinear short-run dynamics in the adjustment process to deviations from long-run equilibrium relations are e.g. contemporaneous price differentials for a certain good. Since it is acknowledged that Jevons's law of one price does not apply intertemporally, researchers have decided to ease conventional restrictions like the assumption of efficient markets. For instance exchange rates under the purchasing power parity in the presence of transaction costs exemplify the necessity of regime-switching dynamics in econometrics, compare Taylor et al. (2001) or Taylor (2001).

However, first advances in nonlinear cointegration refer to Balke and Fomby (1997) who introduced threshold cointegration. According to them error correction requires the disequilibrium error to exceed a critical threshold, implying that price deviations between two locations are corrected by arbitrage only when deviations were sufficiently large. Subsequent extension can be found in Siklos and Granger (1997) or Chen et al. (2005). For particular contributions with respect to testing see Enders and Granger (1998), Lo and Zivot (2001) or Hansen and Seo (2002). If the switch is rather smooth than discrete STR ECMs, brought forward by e.g. Taylor and Peel (2000) or Kiliç (2011), are applied. If the transition between the slowly adjusting 
inner regime and the quickly adjusting outer regimes are associated with small and large price deviations respectively, an exponential STR ECM should be employed. If negative and positive deviations are corrected differently the adjustment process is subject to asymmetric behaviour. In that case a logistic transition function is just appropriate for the adjustment process.

In this paper we propose D-LSTR as an overall generalisation of STR functions. More precisely this work addresses STR-based nonlinear adjustment processes and especially a globally stationary Double-LSTR cointegration process with symmetric behaviour in the outer regimes. The aim is to show that D-LSTR cointegration has better power than other STR functions. We are especially interested in the power results compared to KSS's nonlinear cointegration test based on a globally stationary exponential-STR cointegration alternative.

The rest of the paper is organized as follows. In section 2 the testing framework for the $t$ - and $F$-type test is set up and section 3 the cointegration tests are introduced. Section 4 presents the power results and section 5 concludes.

\section{Model setup}

We start with a nonlinear vector error correction model (VECM) as in KSS, derived from an $(n \times 1)$-vector $\mathbf{z}_{t}=\left(z_{1 t}, \ldots, z_{n t}\right)$, consisting of I(1) stochastic processes being given by

$$
\Delta \mathbf{z}_{t}=\alpha \beta^{\prime} \mathbf{z}_{t-1}+\mathscr{G}\left(\beta^{\prime} z_{t-1}\right)+\sum_{i=1}^{p} \Gamma_{i} \Delta z_{t-i}+\varepsilon_{t}, \quad \text { with } t=1, \ldots, T .
$$

The first and second term on the right hand side represent the linear and nonlinear error correction term. $\alpha_{(n \times r)}$ contains the linear adjustment parameters, that describe the percentaged correction in period $t$, while $\beta_{(n \times r)}$ is the cointegrating vector. The cointegration relation is assumed to be linear which is why the second error correction term simply underlies a nonlinear transformation according to the insinuated nonlinear transition function, $\mathscr{G}(\cdot)$. Concerning the specific transition function $\mathscr{G}(\cdot)$ in our testing approach we will go into detail in the ongoing subsection. For some further explanatory power of the model lagged autocorrelations are included in $\Gamma$, depending on the optimal lag order $p$. The $(n \times n)$ error process $\varepsilon_{t}$ is $\operatorname{iid}(\mathbf{0}, \Sigma)$ with $\Sigma$ being a positive definite matrix. It is assumed that the initial values $\mathbf{Z}_{\mathbf{0}} \equiv\left(\mathbf{z}_{-p}, \ldots, \mathbf{z}_{0}\right)$ are known and $A(z)$ is given by $(1-z) \mathbf{I}_{n}-\alpha \beta^{\prime} z-\sum_{i=1}^{p} \Gamma_{i}(1-z) z^{i}$. If $\operatorname{det} A(z)=0$, then $|z|>1$ or $z=1$ what implies that the number of unit roots equals $n-r$ with $r$ being the quantity of cointegration relations.

Since we intent to analyze at most one conditional long-run cointegration relation the vector $\mathbf{z}_{t}$ is decomposed into $\left(y_{t}, \mathbf{x}_{t}^{\prime}\right)^{\prime}$, the dependent and the explanatory variable respectively. The scalar $y_{t}$ is hereby conditioned by $\mathbf{x}_{t}$ given the past values of $\mathbf{z}_{t}$. Hence we obtain 


$$
\Delta \mathbf{z}_{t}=\alpha u_{t-1}+\mathscr{G}\left(u_{t-1}\right)+\sum_{i=1}^{p} \Gamma \Delta z_{t-i}+\varepsilon_{t}, \quad t=1, \ldots, T
$$

whereby the linear cointegration relation is enclosed in

$$
u_{t}=y_{t}-\beta_{x}^{\prime} \mathbf{x}_{t},
$$

with $\beta_{x} \sim(k \times 1)$ containing the cointegration parameters and $k$ equal to $(n-1)$.

\subsection{Double logistic smooth transition}

In our model setup we presume that the switches between regimes are induced by a second-order logistic smooth transition or double LSTR (D-LSTR) process, originally proposed by Jansen and Teräsvirta (1996), derived from

$$
\mathscr{G}\left(s_{t} ; \gamma, c\right)=\left(1+\exp \left\{-\gamma\left(s_{t}-c_{1}\right)\left(s_{t}-c_{2}\right)\right\}\right)^{-1}, \quad c_{1} \leq c_{2}, \gamma>0 .
$$

$s_{t}$ is the state variable that causes the switch between regimes. Here $s_{t}$ is replaced by the lagged variable of the cointegration relation's error $u_{t-1}$ where the value of $u_{t-1}$ determines if the threshold is met or not. The threshold values $c_{1}$ and $c_{2}$ are chosen to be $c_{1}=-\sqrt{c}$ and $c_{2}=\sqrt{c}$ assuming that $-c_{1}=c_{2}$ holds. Therefore, $\mathscr{G}(\cdot)$ simplifies to

$$
\mathscr{G}\left(s_{t} ; \gamma, c\right)=\left(1+\exp \left\{-\gamma\left(y_{t-1}^{2}-c\right\}\right)^{-1}, \quad \gamma \geq 0,\right.
$$

and a symmetric transition function is obtained. The smoothness parameter $\gamma$ determines the gradual changing strength of adjustment for the changes in regimes. The reason why we propose D-LSTR in contrast to an ESTR function is that the D-LSTR approach features special properties. Firstly D-LSTR can display symmetric and stationary behaviour in the outer regimes once $u_{t-1}<-\sqrt{c}$ or $u_{t-1}>\sqrt{c}$ on the one hand. On the other hand it can display unit root behaviour at the central regime when $-\sqrt{c}<u_{t-1}<\sqrt{c}$. Secondly, it is capable of generating exponentialtype nonlinearity that closely approximates ESTR nonlinearity, when the transition parameter tends to infinity, cf. Sollis (2011), even though the D-LSTR model does actually not nest an ESTR-model. Contingent on the value of $\gamma$ and due to its special properties the D-LSTR function covers not only exponential-type nonlinearity for small and moderate $\gamma$ but nests 3-regime TAR nonlinearity for $\gamma \rightarrow \infty$. Consequently, a self-exciting TAR model is obtained since the state variable equals the transition variable depending on whether the linear combination of $y_{t}$ and $\mathbf{x}_{\mathbf{t}}$ is stationary or not. This means that the switching of the model depends on the cointegratedness of $y_{t}$ and $\mathbf{x}_{\mathbf{t}}$. With respect to the assumptions on $c_{1}$ and $c_{2}$ the outer regimes of this self-exciting TAR model are restricted to be identical.

Furthermore, D-LSTR offers more flexibility concerning the range of the nonsta- 
tionary regime due to the scaling parameter $c$, e.g. Kaufmann et al. (2012). In contrast to D-LSTR a possible drawback of an exponential transition function would be that for $\gamma \rightarrow 0$ and $\gamma \rightarrow \infty$, the model becomes linear, cf. van Dijk et al. (2002). It should be mentioned that unlike the logistic function the second order logistic function is not bounded between $[0,1]$. For finite $\gamma$ the D-LSTR function realises a minimum different from zero, see van Dijk and Franses (2000). In fact, when $\gamma=0$, the D-LSTR function $\mathscr{G}(\cdot)$ reduces to 0.5 and the model becomes linear. For this reason, in our testing approach we propose the transition function

$$
\mathscr{G}\left(u_{t-1} ; \gamma, c\right)=\left[\left(1+\exp \left\{-\gamma\left(u_{t-1}^{2}-c\right)\right\}\right)^{-1}-0.5\right], \quad \gamma>0,
$$

following Teräsvirta (1994), who included -0.5 in order to derive linearity tests. In our case subtracting 0.5 ensures that there is no cointegration at all and therefore enables us to test the problem under consideration, what will be issued in an instant. So far, our partitioned model assembles to

$$
\begin{aligned}
\Delta y_{t} & =\phi u_{t-1}+\rho u_{t-1}\left[\left(1+\exp \left\{-\gamma\left(u_{t-1}^{2}-c\right)\right\}\right)^{-1}-0.5\right]+\omega^{\prime} \Delta \mathbf{x}_{t} \\
& +\sum_{i=1}^{p} \psi_{i}^{\prime} \Delta \mathbf{z}_{t-i}+\varepsilon_{t} \\
\Delta \mathbf{x}_{t} & =\sum_{i=1}^{p} \Gamma_{x i} \Delta \mathbf{z}_{t-i}+\varepsilon_{x t} .
\end{aligned}
$$

Under the assumption that $\phi=\xi-\gamma$ with $\xi<0$ the conditional double logistic STR $\mathrm{ECM}$ for $\Delta y_{t}$ and a marginal vector autoregression model for $\Delta \mathbf{x}_{t}$ is obtained. For further assumptions and details on certain parameter constraints see KSS.

\subsection{Testing problem}

We want to test no cointegration against the alternative of globally stationary DLSTR cointegration. This implies that under the null hypothesis it has to be assured, that there is no cointegration in the process. Nonlinear cointegration is solely embodied via the transition function (5) and (6), which consequently needs to be excluded under $H_{0}$. As $\mathscr{G}(\cdot)$ reduces to 0.5 , when $\gamma=0$, subtracting one half establishes a feasible null hypothesis. This enables us straightforwardly, to formulate the hypotheses as

$$
H_{0}: \gamma=0 \quad \text { vs. } \quad H_{1}: \gamma>0
$$

for testing against globally stationary D-LSTR cointegration. Obviously, $\gamma=0 \mathrm{im}$ plies that $\rho$ and $c$ are not identified under the Null, referred to as the Davies (1987) problem. The stationarity properties of $u_{t}$ are determined by the positiveness of $\gamma$. For solving the cointegration problem and in order to test for the nonlinear cointegration relation we apply the Engle and Granger (1987) residual-based two step 
procedure. At the first stage the residuals $\hat{u}=y_{t}-\hat{\beta}_{x} \mathbf{x}$ are estimated via OLS. At the second stage we expand a first order Taylor series approximation to the smooth transition function due to the non-identification of $\rho$ ( $\rho$ and $c$ ) in the case of a $t$-type test $(F$-type test). The linearisation leads to

$$
T_{1}(\gamma)=0.5+0.25 \gamma\left(u_{t-1}^{2}-c\right)
$$

It might seem more appropriate to use a Taylor expansion of a higher order since it captures the symmetric property far better than the line of the first order. Nevertheless, this implies more terms and respectively more restrictions to be tested, which might result in a loss of power.

Substituting (7) into (6) we obtain the following auxiliary regression

$$
\Delta y_{t}=\delta_{1} \hat{u}_{t-1}+\delta_{2} \hat{u}_{t-1}^{3}+\omega^{\prime} \Delta \mathbf{x}_{t}+\sum_{i=1}^{p} \psi_{i}^{\prime} \Delta \mathbf{z}_{t-i}+e_{t},
$$

where we define $\delta_{1} \equiv \phi-0.25 \rho \gamma c$ and $\delta_{2} \equiv 0.25 \rho \gamma$. In accordance with KSS we assume that $\phi=0$ so that a unit root behaviour around the equilibrium can occur. Imposing $\phi=0$ does not influence the $F$-type test as long as $c \neq 0$. For the case that $c=0$ the test reduces to a $t$-type test.

\section{Cointegration tests}

Setting the switch point $c$ equal to zero finds theoretical justification in many economic and financial applications. Preferably it is utilised in the context of an ESTR function. However, this leads to the following auxiliary regression for the $t$-type test, where $\delta_{1}$ and respectively $\hat{u}_{t-1}$ cancel out

$$
\Delta y_{t}=\delta_{2} \hat{u}_{t-1}^{3}+\omega^{\prime} \Delta \mathbf{x}_{t}+\sum_{i=1}^{p} \psi_{i}^{\prime} \Delta \mathbf{z}_{t-i}+e_{t},
$$

with the corresponding hypotheses

$$
H_{0}: \delta_{2}=0 \quad \text { vs. } \quad H_{1}: \delta_{2}<0 .
$$

The $t$-statistic is given by

$$
t=\frac{\hat{\mathbf{u}}_{-1}^{3^{\prime}} \mathbf{Q}_{1} \Delta \mathbf{y}}{\sqrt{\hat{\sigma}_{N E C}^{2} \hat{\mathbf{u}}_{-1}^{3^{\prime}} \mathbf{Q}_{1} \hat{\mathbf{u}}_{-1}^{3}}}
$$

where $\hat{\mathbf{u}}_{-1}^{3}=\left(\hat{u}_{0}^{3}, \ldots, \hat{u}_{T-1}^{3}\right), \quad \mathbf{Q}_{1}=\mathbf{I}_{T}-\mathbf{S}\left(\mathbf{S}^{\prime} \mathbf{S}\right)^{-1} \mathbf{S}^{\prime}, \quad \mathbf{S}=\left(\Delta \mathbf{X}, \Delta \mathbf{Z}_{-1}, \ldots, \Delta \mathbf{Z}_{-p}\right)$ and $\Delta \mathbf{y}=\left(\Delta y_{1}, \ldots, \Delta y_{T}\right)^{\prime}$.

Assuming that $c \neq 0$ the auxiliary regression is given by (8). As we have two re- 
strictions in the $F$-type test case the corresponding couple of hypotheses for testing for nonlinear cointegration are given by:

$$
H_{0}: \delta_{1}=\delta_{2}=0 \quad \text { vs. } \quad H_{1}: \delta_{1} \neq 0 \text { or } \delta_{2}<0 .
$$

The $F$-type statistic has the form

$$
F_{N E C}=\frac{\left(R S S_{0}-R S S_{1}\right) / 2}{R S S_{1} /(T-3-p)},
$$

where $R S S_{0}$ is the residual sum of squares obtained by imposing the two restrictions given under the null hypothesis, $\delta_{1}=\delta_{2}=0$ and $R S S_{1}$ is the residual sum of squares under the alternative. Since the alternative to a unit root is actually one-sided in the direction of stable roots, like here $\delta_{2}$ is restricted to be less than zero, it might be beneficial to take the one-sidedness of the alternative into account. For this purpose, an approach that incorporates one-sided alternatives can be found in Abadir and Distaso (2007).

In either the $t$-type test or the $F$-type test case will the limiting distribution be nonstandard under the null hypothesis due to the fact that in case of a non-cointegrated relation the series remain nonstationary. Hence, the limiting distributions converge to some functionals of Brownian motions. By similar arguments as in KSS we derive for the $t$-type test

$$
t_{N E C}=\frac{\int B^{3} \mathrm{~d} W}{\sqrt{\int B^{6} \mathrm{~d} \alpha}}
$$

and for the $F$-type test

$$
F_{N E C}=\frac{1}{2}\left[\int B \mathrm{~d} W\right]\left[\begin{array}{l}
\int B^{2} \mathrm{~d} \alpha \int B^{4} \mathrm{~d} \alpha \\
\int B^{4} \mathrm{~d} \alpha \int B^{6} \mathrm{~d} \alpha
\end{array}\right]^{-1}\left[\begin{array}{c}
\int B \mathrm{~d} W \\
\int B^{3} \mathrm{~d} W
\end{array}\right],
$$

where $B$ and $W$ are shorthand notations for $B(\alpha)=W(\alpha)-\mathbf{W}_{x}(\alpha)^{\prime}\left(\int_{0}^{1} \mathbf{W}_{x}(\alpha) \mathbf{W}_{x}(\alpha)^{\prime} \mathrm{d} \alpha\right)^{-1} \times\left(\int_{0}^{1} \mathbf{W}_{x}(\alpha) W_{x}(\alpha) \mathrm{d} \alpha\right)$ where $W(\alpha)$ and $\mathbf{W}_{x}(\alpha)$ defined on $\alpha \in[0,1]$ are independent scalar and $k$-vector standard Brownian motions. For a proof hereof see KSS.

\section{Finite Sample Properties}

In order to examine the power results in dependence of the two major parameters $\gamma$ and $\rho$ we conduct a Monte Carlo study. For this purpose, the model is simplified to a bivariate ECM, where $\beta_{x}$ is assumed to be equal to one and 


$$
\begin{aligned}
\Delta y_{t} & =\lambda \Delta x_{t}+\rho u_{t-1}\left[\left(1+\exp \left\{-\gamma\left(u_{t-1}^{2}-c\right)\right\}\right)^{-1}-0.5\right]+\varepsilon_{t} \\
\Delta x_{t} & =v_{t}, \quad u_{t}=y_{t}-\beta_{x} x_{t} \\
{\left[\begin{array}{l}
\varepsilon_{t} \\
v_{t}
\end{array}\right] } & \sim \operatorname{iid} \mathscr{N}\left(\mathbf{0},\left[\begin{array}{cc}
\sigma_{1}^{2} & 0 \\
0 & \sigma_{2}^{2}
\end{array}\right]\right) .
\end{aligned}
$$

The parameter constellations under investigation are the following:

$\lambda=\{0.5,1\}, \rho=\{-1.0,-0.5,-0.3,-0.1\}, \gamma=\{0.8,1,2,1000\}$, and $\sigma_{2}=\{1,4\}$.

Because $\gamma$ does not only determine the smoothness of adjustment but determines also how present the effect of the nonlinear error correction is, we expect the test to have power finding a nonlinear cointegration relation, when $\gamma$ becomes larger. Therefore, we vary $\gamma$ as is illustrated bellow. In accordance with KSS we investigate the impact of the common factor restriction, $\lambda=1$, for serial correlation in the disturbances. Therefore, we consider different parameter values for $\lambda=\{0.5,1\}$ and also we want investigate the impact of different signal-to-noise ratios and vary $\sigma_{2}^{2}=\{1,4\}$.

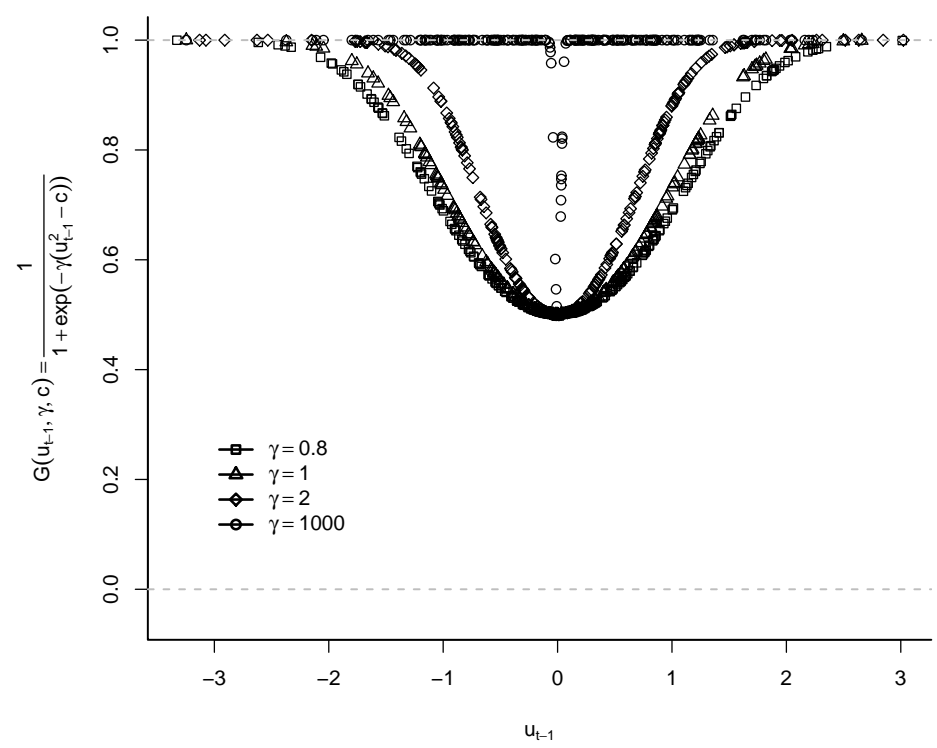

Figure 1: Transition function depending on a varying $\gamma$ with a $c=0$.

As mentioned before, the codomain for the transition probabilities has been scaled down to a half and to $[0.5,1]$ respectively. $\gamma<1$ are frequently chosen values in the ESTR context, which is why $\gamma$ is set equal to 0.8 , compare the squared line. 
The triangled and hashed lines show a still very smooth transition whereas the circled line graphs a very fast transition at $\gamma=1000$. Here the speedy transition results in a 3-regime TAR approximation.

$\rho$ determines how present the nonlinear cointegration is which is why we expect a drop in the power for a sinking $\rho$. The values for $\lambda$ are taken from KSS.

In the following table the power results for our $t$ - and $F$-type test are presented. Additionally we compare these results to a linear cointegration test, wherefore we conducted the Johansen procedure on the globally stationary D-LSTR process, cf. Johansen $(1988,1991)$ in order to discriminate between a nonlinear and a linear cointegation test. The table provides the power results for all possible combinations of the before mentioned parameter constellations $\sigma_{2}, \rho, \gamma$, and $\lambda$. The results are displayed in Table 1 and Figure 2. 


\subsection{Power Results}

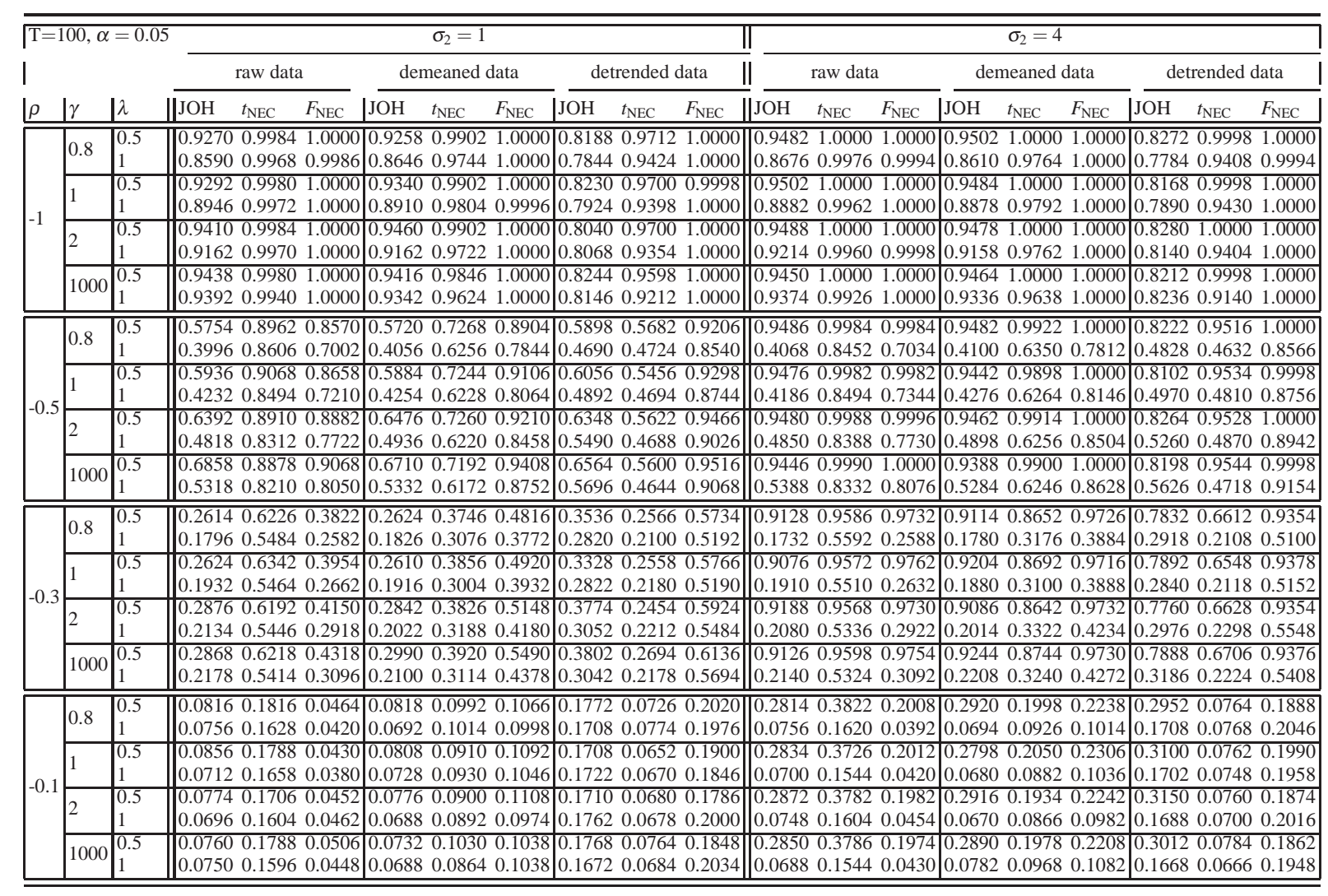

Table 1: Power results for varying parameter constellations of $\left\{\sigma_{2}, \lambda, \gamma, \rho\right\}$. 
One can recognize a clear power loss for $\rho>-1$ when $\sigma_{2}=1$. In case that $\sigma_{2}=4$ the power loss begins for $\rho>-0.3$ for raw and demeaned data and for detrended data at $\rho>-0.5$. A power loss for a sinking magnitude of $\rho$ is quite plausible as $\rho<1$ determines how present cointegration is and thus ensures global stationarity. The power values within a particular block of the same kind of data and for the same $\rho$ are however alike. Apparently the transition speed does not make a big difference to the power when $\gamma$ varies among $\{0.8,1,2,1000\}$. The power gain for a faster transition is marginal. This finding might be due to the possibly low amount of observations in the outer regimes.
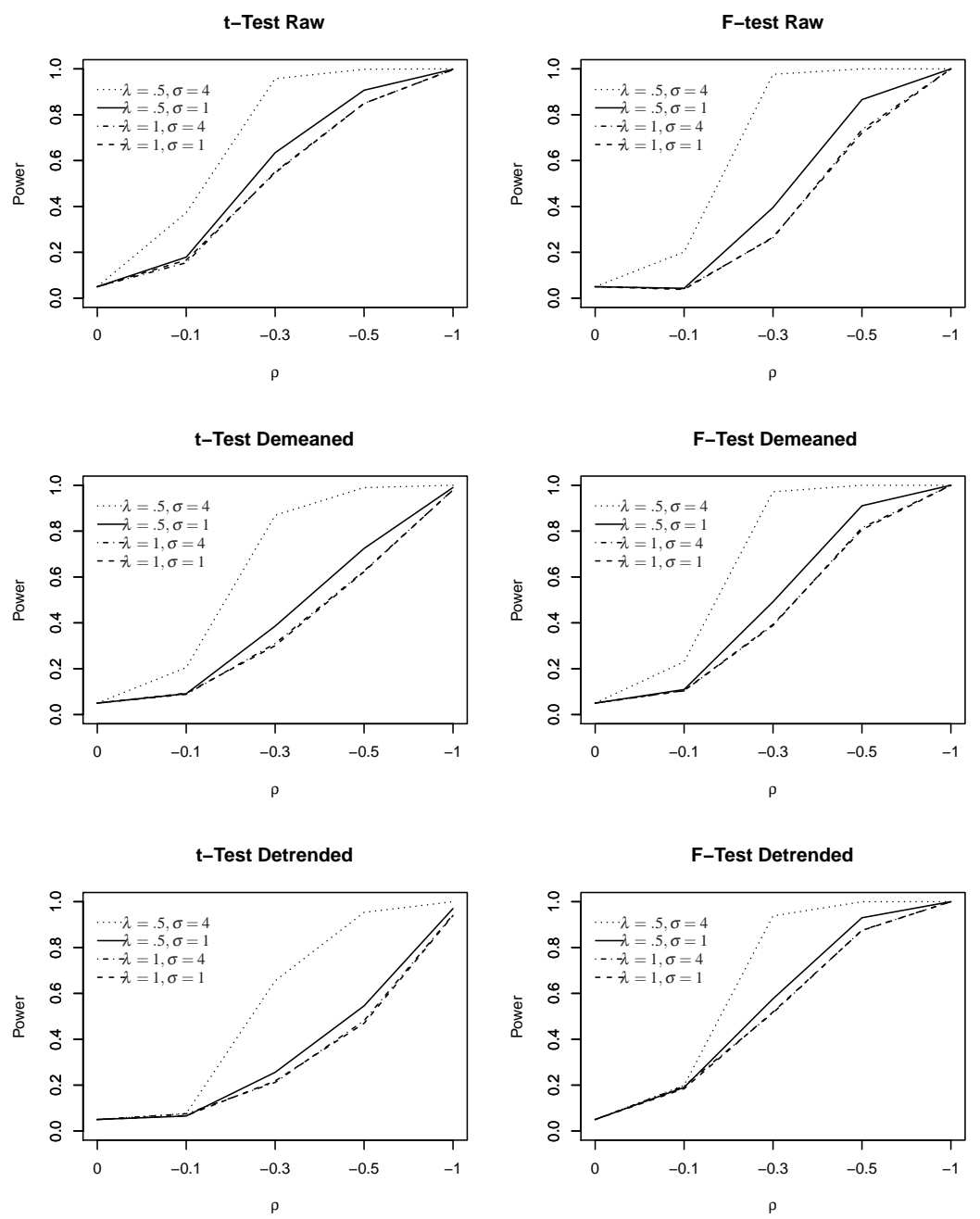

Figure 2: Power results for the $t$ - and $F$-type test for $\gamma=1$. 
It is interesting to observe that the $F$-type test gains power when the data is demeaned or detrended whereas the $t$-type test looses power. Regarding the graphs it can bee seen, that the power for $\lambda=0.5$ dominates the power results for $\lambda=1$ for both tests and all kinds of data sets and moreover, increases with the variance of the innovations in the regressor $x$. This finding is analogue to KSS, where the nonlinear tests have superior power when the common factor restriction is violated, which is due to the increased correlation with the regression error, see KSS. As expected Johansen's linear cointegation test is beaten by the nonlinear cointegration tests $(t$ and $F$ ) for all different kinds of data sets, see Table 1.

\section{Conclusion}

Our proposed D-LSTR function that nests discontinuous adjustment behaviour and is also able to mimic ESTR behaviour has better power than a comparable linear cointegration test. Even though it can be stated for the $t$ - and $F$-type test that there is a significant power drop for the case when $\rho \geq-0.3$ implying that the cointegration relation is quite weakly present in the process, we can nevertheless conclude that our extension of the KSS testing procedure offers reasonable power results. Compared to the $t$-type test the $F$-type test provides even slightly better power results.

In addition to our approach it would be interesting to further discriminate between different cases for $c \neq 0$, what meant a wider inner regime of nonstationarity. 


\section{References}

Abadir, K. and Distaso, W. (2007). Testing joint hypotheses when one of the alternatives is one-sided. Journal of Econometrics, 140:695-718.

Balke, N. and Fomby, T. (1997). Threshold cointegration. International Economic Review, 38:627-645.

Bierens, H. and Martins, L. (2010). Time-varying cointegration. Econometric Theory, 26:1453-1490.

Chen, L.-H., Finney, M., and Lai, K. (2005). A threshold cointegration analysis of asymmetric price transmission from crude oil to gasoline prices. Economic Letters, 89:233-239.

Davies, R. (1987). Hypothesis testing when a nuisance parameter is present only under the alternative. Biometrika, 74:33-43.

Dufrénot, G., Mathieu, L., Mignon, V., and Péguin-Feissolle, A. (2006). Persistent misalignments of the european exchange rates: Some evidence from non-linear cointegration. Applied Econometrics, 38:203-229.

Enders, W. and Granger, C. (1998). Unit-root tests and asymmetric adjustment with an example using the term structure of interest rates. Journal of Business \& Economic Statistics, 16:304-311.

Engle, R. and Granger, C. (1987). Co-integration and error correction: Representation, estimation, and testing. Econometrica, 55:251-276.

Granger, C. (1981). Some properties of time series datat and their use in econometric model specification. Journal of Econometrics, 16:121-130.

Hansen, H. and Seo, B. (2002). Testing for two-regime threshold co-integration in vector error-correction models. Journal of Econometrics, 110:293-318.

Jansen, E. and Teräsvirta, T. (1996). Testing parameter constancy and super exogeneity in econometric equations. Oxford Bulletin of Economics and Statistics, 58:735-763.

Johansen, S. (1988). Statistical analysis of cointegration vectors. Journal of Economic Dynamics und Control, 12:231-254.

Johansen, S. (1991). Estimation and hypothesis testing of cointegration vectors in gaussian vector autoregressive models. Econometrica, 59:1551-1580.

Kapetanios, G., Shin, Y., and Snell, A. (2006). Testing for cointegration in nonlinear smooth transition error correction models. Econometric Theory, 22:279-303.

Kaufmann, H., Kruse, R., and Sibbertsen, P. (2012). A simple specification procedure for the transition function in persistent nonlinear time series models. Discussion paper, Leibniz University Hannover.

Kiliç, R. (2011). Testing for co-integration and nonlinear adjustment in a smooth transition error correction model. Journal of Time Series Analysis, 32:647-660.

Lo, M. and Zivot, E. (2001). Threshold co-integration and non-linear adjustment to the law of one price. Macroeconomic Dynamics, 5:533-576.

Maki, D. (2012). Detecting cointegration relationships under nonlinear models: Monte carlo analysis and some applications. Empirical Economics, pages 1-21. 
Nedeljkovic, M. (2011). Testing for smooth transition nonlinearity adjustments of cointegrating systems. Department of Economics, Warwick Economic Research Paper No. 876.

Seo, M. (2004). Cointegration test in the threshold cointegration model. Manuscript, University of Wisconsin-Madison, Department of Economics.

Shi, X. and Phillips, P. (2012). Nonlinear cointegrating regression under weak identification. Econometric Theory, 28:509-547.

Siklos, P. and Granger, C. (1997). Regime sensitive cointegration with an application to interest-rate parity. Macroeconomic Dynamics, 1:640-657.

Sollis, R. (2011). Testing the unit root hypothesis against TAR nonlinearity using STAR-based tests. Economic Letters, 112:19-22.

Taylor, A. (2001). Potential pitfalls for the purchasing power parity puzzle? Sampling and specification biases in mean reversion tests of the law of one price. Econometrica, 69:473-498.

Taylor, A. and Peel, D. (2000). Nonlinear adjustment, long-run equilibrium and exchange rate fundamentals. Journal of International Money and Finance, 19:3353.

Taylor, M., Peel, D., and Sarno, L. (2001). Nonlinear mean reversion in real exchange rates: Toward a solution to the purchasing porwer parity puzzles. International Economic Review, 42:1015-1042.

Teräsvirta, T. (1994). Specification, estimation, and evaluation of smooth transition autoregressive models. Journal of the American Statistical Association, 89:208218.

van Dijk, D. and Franses, P. (2000). Nonlinear error-correction models for interest rates in the netherlands. Non-linear Econometric Modelling in Time Series Analysis (W. Barnett, DF Hendry, S. Hylleberg, T. Teräsvirta, D. Tjøstheim and AW Würtz, eds.), Cambridge: Cambridge University Press (2000), pages 203-227.

van Dijk, D., Teräsvirta, T., and Franses, P. (2002). Smooth transition autoregressive models - A survey of recent developments. Econometric Reviews, 21:1-47.

Zhang, L. (2013). Revisiting the empirics of inflation: A smooth transition error correction approach. Economics Letters, 119:68-71. 